\title{
Risk factors of ischemic cerebral vascular accident
}

\author{
N.H. Ngoc and N.Q. An \\ Phu Tho General Hospital, Viet Nam \\ Corresponding author: N.Q. An \\ E-mail: longdangtrang@gmail.com \\ Genet. Mol. Res. 20 (1): gmr18742 \\ Received December 30, 2019 \\ Accepted April 20, 2020 \\ Final Revision January 25, 2021 \\ Published March 29, 2021 \\ DOI http://dx.doi.org/10.4238/gmr18742
}

\begin{abstract}
Stroke is one of the leading causes of death both globally and in Southeast Asia, which is confirmed by high values: 5 million and 500 thousand deaths annually, respectively. The situation in Vietnam is also critical. We have systematized and studied the risk factors in patients with ischemic stroke in Phu Tho Province General Hospital. This was a prospective cohort study of 192 patients. Transient ischemic attack was described in $13 \%$ of all cases, and the risk of progression to severe disease or death had an odds ratio (OR) $=5.2 ; 63.5 \%$ of all patients with hypertension had a risk of severe complications or death with OR 3.2. Previous cardiovascular events were found during the history of the disease in $28.1 \%$ of patients. Hyperlipidemia had a significant prevalence among the patients; the risk of exacerbation was $\mathrm{OR}=2.6$. Tobacco smoking and alcohol abuse also had a high prevalence among patients $(72.6 \%)$. The risk of death among smokers and alcohol abusers was 2.4 times higher compared with patients without bad habits. Ischemic cerebrovascular accident is one of the most common health problems in Vietnam and needs more attention.
\end{abstract}

Key words: Risk factors analysis; Ischemic stroke; Hemorrhagic stroke; Ischemic cerebrovascular accident; Stroke risk factors 


\section{INTRODUCTION}

Cerebrovascular accident has a high prevalence and frequency and is a global health problem. Thus, according to the latest data, about a million cases of cerebrovascular accidents are observed in Europe every year (Donkor, 2018). Current research shows that in the USA, one stroke happens every 40 seconds and it is approximately equal to 800 thousand accidents per a year (Nakajima and Chester, 2019). In Asia, the incidence of stroke is different between different countries. For example, in Japan the stroke rate is $303 / 100,000$ of the population; in China this value equals $115.6 / 100,000$, while it is $370 / 100,000$ in the Beijing population alone (Nguyen Minh Hien, 2013).

Along with acute cardiovascular events and malignant neoplasms, stroke is one of the leading causes of mortality in the adult population worldwide, despite significant progress in the diagnosis and preventive measures of this pathology. Stroke types include acute ischemia (AIS) and acute hemorrhagic (AHS) stroke. AIS is characterized by a higher frequency and prevalence, which is about $80-85 \%$.

In Vietnam, the researcher Le Van Thanh investigated 2962 stroke patients: 40.4\% of them had AHS. In another study by Hoang Khanh in Hue city, the AHS accounted for 39.4\% (Trinh Viet Thang, 2011).

An average life expectancy in Vietnam has increased due to improved socialeconomic conditions; but unfortunately, at the same time more strokes are observed (Dao Thi Bich Hoa, 1996; Dang Quang Tam, 2005). For AIS, data have accumulated from a significant number of previous studies, but the possible correlation of the risk factors with this pathology has not been studied.

It should be noted that these evaluations did not consider the presence of concomitant diseases, even though patients were initially in general departments: cardiological, therapeutic, and neurological. Thus, studies of such design have not been conducted before, have scientific novelty, are relevant and appropriate for studying the actual state of AIS and preparing resources for the diagnosis, treatment, and prevention of AIS. It is important to consider that AIS and AHS have similar risk factors, but there are some notable differences. Both types of stroke are characterized by non-modifiable and modifiable stroke risk. Non-modifiable risks similar for both types are age, gender, race and ethnicity (Boehme et al., 2017). Similar modifiable factors are Hypertension (HTN), Transient Ischemic Attack (TIA), genetic heritage, blood pressure, bad habits (alcohol, tobacco etc.) (Bhatt et al., 2008). Different factors that cause ischemic stroke are heart disease, hyperlipidemia, physical inactivity, and diabetes (Boehme et al., 2017).

We examined the risk factors for AIS in Phu Tho Provincial Hospital.

\section{MATERIAL AND METHODS}

Between October 2014 and October 2016, more than 190 AIS patients received medical care in Phu Tho provincial general hospital. We retrospectively studied data contained in medical records of patients. The World Health Organization (WHO) diagnostic criteria of the AIS in 1990: sudden symptoms (minutes to hours, days) which prolong more than 24 hours, with focal symptoms (due to impaired arteries) without a correlation to a trauma (Roth et al., 2015). The Brain Magnetic Resonance Imaging (MRI) showing at least one of the two signs: Homogeneous intensity on T1W, a T2W hyperintensity in the 
subcortical area and loss of differences between the parenchyma and cortex. A hypodense signal on T1W and a hyperintense signal on T2W (Hoang Duc Kiet, 2004). The Transient Ischemic Attack (TIA), ischemic stroke in a patient with the prehistory of head trauma or blunt trauma. Comorbidity of encephalitis, meningitis or brain tumor; ischemic stroke converted to hemorrhage; relapsed ischemic stroke or normal finding on MRI. Statistical Analysis: Software SPSS 18.0 and STATA 10.0

The study design and the content of the patient's informed consent were approved at a meeting of the institutional expert committee on ethics and bioethical examination.

\section{RESULTS}

The following section shows the statistical data. An investigation of the issue analyzed the data of 192 patients. All respondents agreed on the process of the data in accordance with the ethical standards of the responsible committee on human experimentation (institutional or regional) and with the Helsinki Declaration of 1975, as revised in 2000 .

\section{A pre-history of an addiction}

As it has been mentioned above, alcohol and tobacco are associated with Ischemic Cerebrovascular Accident (ICA) (Han and Berry, 2019). The statistical data of the main factors that reveals the precursors of the ischemic disease are shown in Table 1 .

\begin{tabular}{|c|c|c|c|c|c|}
\hline \multirow{2}{*}{ History } & \multicolumn{2}{|c|}{ Group II $(n=64)$} & \multicolumn{2}{|c|}{ Group I $(n=128)$} & \multirow{2}{*}{$\begin{array}{l}n(\%) \\
(n=192)\end{array}$} \\
\hline & $\mathbf{n}$ & $\%$ & $\mathbf{n}$ & $\%$ & \\
\hline \multirow{3}{*}{$\begin{array}{l}\text { Alcohol abuse } \\
\text { Tobacco addiction } \\
\text { Alcohol and tobacco } \\
\text { addiction }\end{array}$} & 22 & 34.4 & 27 & 21.1 & $49(25.8 \%)$ \\
\hline & 27 & 42.2 & 26 & 20.3 & $52(57.4 \%)$ \\
\hline & 17 & 25.5 & 21 & 16.4 & $37(19.4 \%)$ \\
\hline
\end{tabular}

As it has been observed in Table 1, it is possible to conclude that in both groups the participation of alcohol abuse equals $25.8 \%$. Tobacco addiction was slightly more frequent. The rate of both addictions was lower, involving $19.4 \%$ of the patients.

\section{Other pre-medical history}

During the research, other diseases were determined including: HTN, diabetes, heart diseases and TIA (Table2).

Diagnostic results showed that a major risk rate corresponds to a patient group of heart diseases and equals to $28.1 \%$. The intermediate and lower rates correspond to the patients with diabetes and the TIA. The critical risk factoring of $63.5 \%$ is associated 
with the HTN. The following results show that in Vietnam a real quantity of people with HTM disease is higher than it had been mentioned in statistical data received through the 2005 National Adult Obesity Survey by the Do Phi Phuong Ha (Do, 2014). A patient consultation was conducted to initiate lifestyle modification, promotion of physical exercises and healthy food during a day. In Table 3, a pressure measurement of the HTN group is shown.

Table 2. Pre-medical history showing role of risk factors.

\begin{tabular}{llllll}
\hline History & N & \% & n & \% & \\
\hline Hypertension (HTN) & 52 & 80.2 & 70 & 54.7 & $122(63.5 \%)$ \\
Diabetes & 12 & 19.8 & 18 & 14.1 & $30(15.6 \%)$ \\
Heart diseases (Heart failure. AF. & 21 & 33.3 & 33 & 25,8 & $54(28.1 \%)$ \\
Mitral valvestenosis) & 18 & 28.6 & 7 & 5.4 & $25(13.0 \%)$ \\
Transient ischemic attack & & & & \\
\hline
\end{tabular}

Table 3. Blood pressure measurement in patients with hypertension.

\begin{tabular}{llllc}
\hline Blood pressure (BP) & Minimum & Maximum & Mean & SD \\
\hline Systolic BP (mmHg) & 140 & 180 & 152 & 10.2 \\
Diastolic BP (mmHg) & 70 & 120 & 94 & 8.9 \\
Years of hypertension & 1 & 20 & 7 & 4.7 \\
\hline
\end{tabular}

Also, all patients from the first and second groups were qualified based on number of risk factors (Table 4).

Table 4. Patient classification based on number of risk factors.

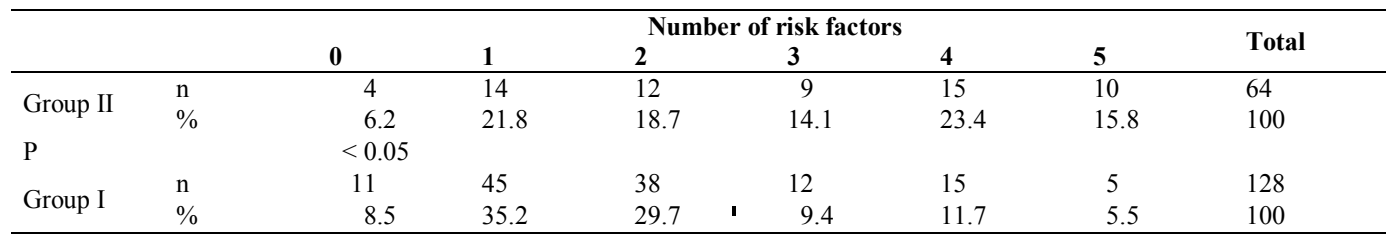

The predictive value of some risk factors: An expression of the likelihood oof disease was based on investigation, which correlates with the positive or negative test results of the diseases is shown in Tables 5, 6 and 7.

Table 5. Patient distribution based on disease severity.

\begin{tabular}{lll}
\hline & Number of patients, $\mathbf{n}$ & Ratio, \% \\
\hline Group I (mild and medium) & 128 & 66.7 \\
Group II (severe - death) & 64 & 33.3 \\
Total amount & 192 & 100 \\
\hline
\end{tabular}


Table 6. Predictive value for ischemic stroke development based on pre-medical historical risk factors.

\begin{tabular}{llll}
\hline Diseases & Yes & No & Total \\
\hline Hypertension & & $12(19.0 \%)$ & 64 \\
Group II & $52(81.0 \%)$ & $57(44.6 \%)$ & 128 \\
Group I & $71(55.4 \%)$ & $\mathrm{p}=0.009$ \\
OR=3.2 & $95 \% \mathrm{CI}=1.3-7.8$ & & \\
$\begin{array}{l}\text { Heart diseases (Heart failure. AF. mitral valve stenosis) } \\
\text { Group II }\end{array}$ & $22(34.3 \%)$ & $42(65.6 \%)$ & $64(100 \%)$ \\
Group I & $34(26.5 \%)$ & $94(73.5 \%)$ & $128(100 \%)$ \\
OR=26 & $95 \% \mathrm{CI}=1.1-6.1$ & & $\mathrm{p}=0.034$ \\
Diabetes & & & \\
Group II & $13(20.3 \%)$ & $51(79.7 \%)$ & $64(100 \%)$ \\
Group I & $19(14.8 \%)$ & $109(85.2 \%)$ & $128(100 \%)$ \\
OR=2.2 & $95 \% \mathrm{CI}=0.9-5.1$ & & $\mathrm{p}=0.073$ \\
\hline
\end{tabular}

Table 7. Predictive values of magnetic resonance imaging (MRI) findings.

\begin{tabular}{|c|c|c|c|}
\hline & \multicolumn{2}{|c|}{ Size of ischemic area } & \multirow{2}{*}{ n (\%) } \\
\hline Severity & $<3 \mathrm{~cm}$ & $\geq 3 \mathrm{~cm}$ & \\
\hline Group II & $27(42.2 \%)$ & $37(57.8 \%)$ & $64(100 \%)$ \\
\hline Group I & $81(63.2 \%)$ & $47(36.8 \%)$ & $128(100 \%)$ \\
\hline $\mathrm{OR}=3.4$ & \multicolumn{2}{|c|}{$95 \% \quad \mathrm{CI}=1.5-7.4$} & $\mathrm{p}=0.002$ \\
\hline Severity & \multicolumn{2}{|c|}{ Number of ischemic areas } & n (\%) \\
\hline Group II & $48(75 \%)$ & $16(25.0 \%)$ & $64(100 \%)$ \\
\hline Group I & $94(73.5 \%)$ & $34(26.5 \%)$ & $128(100 \%)$ \\
\hline $\mathrm{OR}=0,7$ & $95 \%$ & & $\mathrm{p}=0.536$ \\
\hline
\end{tabular}

\section{DISCUSSION}

As a result of our study, we were able to identify etiological risk factors for ischemic cerebrovascular pathology, such as tobacco smoking and alcohol abuse, persistent high blood pressure, diabetes mellitus, cardiovascular pathology and a history of TIA.

\section{Tobacco addiction}

Tobacco smoking tends to increase in prevalence in modern society. Among the countries in which smoking has the highest prevalence, Vietnam is included, as tobacco smoking is widespread among more than half of the male population. Strong direct correlations between tobacco smoking and stroke have been found in various studies. We found that about $27 \%$ of patients in our study were smokers (Table 1), which was higher than the values obtained in the observations of Phan Thi Huong (16\%), and Dinh Van Thang (18\%).

In patients with tobacco smoking, there was a high risk of sudden cardiovascular death in AIS with $\mathrm{OR}=2.4(\mathrm{p}=0.044$; CI: 1.02-5.6). The results of this study do not contradict the data obtained in the studies of other Vietnamese and foreign authors. Thus, in the Le Quang Cuong study, it was shown that smoking among women increased the risk of developing AIS by 1.9 times and 2.5 times in men. In a study by Wolf P.A et al. (1988) 
showed that smoking increases the risk of stroke by about $40 \%$ in men and $60 \%$ in women. Research has shown that having severe nicotine addiction (smoking more than 40 cigarettes a day) doubles the risk of stroke compared to people with mild nicotine addiction (smoking less than 10 cigarettes a day). Thus, there is significant evidence that smoking is a significant risk factor for the development of AIS. (Bui My Hanh et al., 2019).

\section{Alcohol abuse}

More than $25.8 \%$ of the study population is alcoholic which was similar to Le Ngoc Trong (26.9\%) and Dang Quang Tam (31\%); and higher than Pham Thi Thu Ha (6.6\%) and Phan Thi Huong (7\%) (Wolf P.A. et al., 1988; Phan Thi Huong, 2004; Dang Quang Tam, 2005). The odds ratio of alcohol abuse affecting disease severity and death was 2.5 ( $p=$ $0.038, \mathrm{CI}=1.1-6.0$ ) compared to non-alcohol use. Many other studies showed the role of alcohol in the severity of stroke. Zhang's study in China found that people who consumed high volumes of alcohol had a 1.9 times higher risk of stroke than others (Zhang et al., 2014). Mukamal et al. (2003) observed alcohol addicts during 14 years expressed that the habit of drinking 10-29 grams of ethanol a day, 3-4 days a week induced the lowest risk of stroke whereas people who consumed more than two glasses of whiskey a day had the highest risk. Le Quang Cuong reported that if ethanol consumption increased $10 \%$ in the population, it would raise the stroke morbidity up to $29 \%$ and mortality up to $16 \%$ (Nguyen and Hoang, 2018).

Alcohol abuse is associated with an increased risk of hypertension, increased platelet aggregation, and cardiac arrhythmias such as atrial fibrillation. All of the above factors are also risk factors and etiological factors for AIS.

\section{Hypertension (HTN) history}

In accordance with the results of the investigation, HTN was seen in $63.5 \%$ of the population that was higher than Dao Thi Bich Hoa (40\%) (Dao Thi Bich Hoa, 1996). This result from the HTN became more popular, especially in developed countries. In a report by the WHO in 2000 year it is estimated that 972 million people had the HTN and this number was predicted to be 1,5 trillion people in 2025 (World Health Organization, 2003). In the United States of America, the medical system is considered the best in the world and only $77.6 \%$ people recognized the HTN. Since the result was collected by asking the patients directly or their family member so that the actual number of the HTN in the study population, it is probably much higher than the $63.7 \%$ that was reported.

Other studies show that both systolic and diastolic blood pressure independently induced different types of stroke (Webb et al., 2018). It had been found that systolic BP higher than $160 \mathrm{~mm} \mathrm{Hg}$ and the BP diastolic is higher than $95 \mathrm{~mm} \mathrm{Hg}$ could in 3.1 times increase the risk of the AIS in men and 2,9 times in women. If the BP systolic stayed in the range of 140 to $159 \mathrm{~mm} \mathrm{Hg}$ and the $\mathrm{BP}$ diastolic stayed in the range of 90 and $94 \mathrm{~mm} \mathrm{Hg}$ would raise the risk of stroke up to 50\% (Ngo Duc Vuong, 2010).

The longest duration with the HTN, the more severe symptoms of stroke patients could suffer. In our study, the mean duration of the HTN is 7 years. Consequently, chronic HTN patients ought to pay a higher warning attention to the AIS. In accordance with these findings, the HTN put the AIS patient at the risk of getting worse or even death 3,2 times 
higher than non-HTN with the CI: $1.3-7.8$ and $\mathrm{P}=0.009$ ). Other authors estimated that a $10 \mathrm{~mm} \mathrm{Hg}$ reduction in systolic BP in adults would decrease $30 \%$ in the risk of death due to heart disease and also cause a drop of $40 \%$ in the risk of mortality due to stroke. Hence, early recognition and regular treatment of the HTN and community education for this issue are extremely necessary. However, even in the United States of America, only $67.9 \%$ did so, and only $44.1 \%$ of those were tightly controlled (Webb et al., 2018). So, the difficulty in HTN management does not just belong to developing countries, but also involves the developed ones.

\section{Diabetes mellitus}

There have been numerous investigations worldwide showing that diabetes mellitus or diabetes is a crucial risk factor for atherosclerosis. It played an important role in embolism appearance and the AIS. In the United States during 1976-1980, diabetes patients had a risk of AIS from 2.5 to 4 times higher than people who didn't have diabetes (Deshpande et al., 2008). In the context of the Honolulu Cardiovascular Program, which examined the relationship of carbohydrate metabolic disorders to cardiovascular disease, diabetes increased the risk of ischemic disease threefold compared to individuals without carbohydrate metabolic disorders. In another investigation Framingham, although the most influence of diabetes was on microvascular diseases, although that still had a bad effect on coronary and cerebral vascular (Wolf et al., 1988).

In our study, the odd ratios of diabetes on AIS was 2.2. It had been suggested that diabetes could increase the risk of getting the AIS or being worse for AIS, however the nonsignificant $p$ value indicates that we would need further study to check this correlation. In the AIS population, diabetes accounted for $15.6 \%$, which was higher than previous studies such as that of Phan Thi Huong (2004), who reported 6.2\%. One of the alleged reasons has been the increase in the number of people with diabetes in the country in recent years. The sharp increase in the number of patients with diabetes is associated with improper overnutrition and physical inactivity as a result of an increase in living standards. The sharply elevated diabetes rates are probably due to a higher standard of living, and a resulting imbalance between nutrition and physical activity. These should be considered as factors to help reduce diabetes and the resulting AIS morbidity.

\section{Previous Heart Disease}

In our study, $28.1 \%$ of patients had previous heart diseases similar to result from Dang Quang Tam 21.2\%, Dinh Van Thang 6.5\%, and Nguyen Xuan Than 6.1\%. Those patients had an increased risk of severe and death with $\mathrm{OR}=2.6(\mathrm{CI}=1.1-6.1 ; \mathrm{P}=0.034)$ (Dang Quang Tam, 2005). This factor had a high predictive value with $\mathrm{P}<0.05$. In a patient with pre-exist cardiovascular diseases, the risk of the AIS was higher and when they suffered from AIS, the possibility of deterioration was higher because cardiovascular conditions could appear embolism from the heart and reduced peripheral and cerebral perfusion. The Atrial Fibrillation (AF) was a common impairment and was a strong risk of the AIS, a well-cared for the AF therefore would prevent AIS's appearance effectively.

A Framingham study showed that there was a clear elevation of the AIS in the AF patients: $1.5 \%$ at the age of $50-59$ and $23.5 \%$ at the age of $80-89$. The author also impressed 
that $8 \%$ of men and $11 \%$ of women could have AIS within 6 years after an acute Myocardial Infarction (MI).1 Besides the AF, other cardiovascular conditions such as dilated Myocardiopathy (DMC), Mitral Valve Prolapse (MVP), Mitral Valve Stenosis (MVS), Heart Failure (HF), artificial heart valve, acute endocarditis and other congenital heart defects could induce the AIS with different risk level.

Our study did not focus on the detailed diagnosis of heart diseases due to the small number of this group. Furthermore, heart disease has been just a limited part between other important factors needed to be investigated. However, from received limited data, the actual information from the patients who had previous heart diseases did improve the belief and efficacy in the treatment of AIS patients.

\section{Previous Transient Ischemic Attack}

The TIA is an important factor influenced on the risk of the AIS but also the severity of AIS, especially the death risk in the first few days after the AIS on set. The TIA was combined with neurological deficits that existed only within 24 hours and recovered completely, but tended to relapse multiple times later. However, the TIA highly warned about a widespread atherosclerosis and it easily became worse, which could result in the AIS and Multifocal ischemic stroke. In Fleming et al. (2015) study, in the first month, 8\% of the TIA patients suffered from actual AIS, $20 \%$ of these patients had a risk of acute MI, stroke or sudden death in the first 12 months.

In our study it was found that the TIA equals $13.0 \%$. It is similar to Le Thi Hoa Binh 12.8\%, Le Van Thinh 11\% and Dinh Van Thang 8.9\% (Le Van Thinh, 2004). When a patient had the TIA, risk of the AIS severe was highest with $\mathrm{OR}=5.2, \mathrm{p}=0.004$ ). So then, if the patient has previous TIA, patients would risk becoming severely ill or dying 5.2 times higher than patients without the TIA before. The TIA was a clear sign of "near" obstruction in the cerebral vascular system, which raised an important alarm of further poor-prognosis complications after.

In the studies of Fleming, the TIA had the highest risk of the AIS severe compared to all other risk factors such as the HTN, diabetes and heart diseases (Fleming et al., 2015). Because of this, TIA treatment could help to prevent the AIS in the future and was considered more crucial than reperfusion therapies after AIS happened already. From those results are strongly recommended investigating and closely observe the TIA signs to achieve an adequate diagnosis and treatment. Therefore, it could prevent poor-outcome, complications and reduce the AIS severe or death.

\section{Total Number of Risk factors in a patient}

In the mild and medium group, patients had only one or maximum two risk factors while in the severe or death group most patients had at least three risk factors, the difference was significant. Therefore, the more risk factors a patient had previously, the more severe symptoms patients could suffer. In the study of Le Thi Thanh Tuyen and Le Tu Phuong Thao, most patients had two risk factors, rarely worn, especially many patients brought three or more risk factors (Le Thi Thanh Tuyen, 2010). This rang an alarming notice because uncontrolled hyperlipidemia, the HTN, diabetes would contribute directly to the severity of the AIS. Each factor had a special effect on each other. For example, patients 
with a hyperlipidemia tend to have atherosclerosis and the HTN whereas uncontrolled diabetes surely increased atherosclerosis and therefore increased the risk of ischemic stroke.

\section{CONCLUSIONS}

The research shows that with the increasing number of diseases such as diabetes, hypertension, various heart diseases, as well as bad habits and inactive lifestyles can lead to the ICA. As it had been shown in our analysis, with more risk factors patient are more likely to die from the disease.

The TIA was found in $13.2 \%$ of patients which could progress to a severe group with $\mathrm{OR}=5.2$. Hypertension was seen in $63.7 \%$ patients and had a risk of being severe 3.2 times higher than normotensive cases. Patients with heart diseases contributed $28.4 \%$ of the population and risk of getting worse with $\mathrm{OR}=2.6$. The highest rate of hyperlipidemia was caught with risk of deterioration with $\mathrm{OR}=2.6$ ).

Cigarettes and alcohol abuse was $72.6 \%$; smoke addiction had a risk of being worse with $\mathrm{OR}=2.4$ while the risk of alcohol abuse was with $\mathrm{OR}=2.5$. The problem is actual and needs further investigation.

\section{ACKNOWLEDGMENTS}

The authors are grateful to Phu Tho General Hospital for financial support.

\section{CONFLICTS OF INTEREST}

The authors declare no conflict of interest.

\section{REFERENCES}

Bhatt VR, Parajuli N, Mainali NR, Sigdel S, et al. (2008). Risk factors of stroke. J. Inst. Med. 30(3): 37-41.

Boehme AK, Esenwa C and Elkind MS (2017). Stroke risk factors, genetics, and prevention. Circ. Res. 120(3): 472-495. DOI: $10.1161 /$ CIRCRESAHA.116.308398.

Bui MH, Le QC, Nguyen TS, et al. (2019). Determination of Risk Factors for Venous Thromboembolism by an Adapted Caprini Scoring System in Surgical Patients. J. Pers. Med. 9(3): 1-9. DOI: 10.3390/jpm9030036.

Dang QT (2005). Study on several stroke epidemiological features. PhD thesis in Medicine, Hanoi Medical University.

Dao TBH (1996). Comment of Clinical-subclinical features of ischemic stroke in people older than 45 years old. $\mathrm{PhD}$ thesis, Hanoi Medical University.

Deshpande AD, Harris-Hayes M and Schootman M (2008). Epidemiology of Diabetes and Diabetes-Related Complications. Phys Ther. 88(11): 1254-1264. DOI: 10.2522/ptj.20080020.

Do HTP (2014). Hypertension in Vietnam: prevalence, risk groups and effects of salt substitution. Wageningen University, p. 160.

Donkor ES (2018). Stroke in the $21^{\text {st }}$ Century: A Snapshot of the Burden, Epidemiology, and Quality of Life. Stroke Res Treat. 2018: 3238165. https://doi.org/10.1155/2018/3238165

Fleming S, Atherton H, McCartney D, Hodgkinson J, et al (2015). Self-screening and Non-physician screening for hypertension in communities. Am. J. Hypertens. 28: 1316-1324. DOI:10.1093/ajh/hpv029.

Han S and Berry J (2019). What to know about ischemic stroke. Med. News Today. Retrieved from https://www.medicalnewstoday.com/articles/318098 (accessed 28 March 2020).

Hoang DK (2004). Diagnostic imaging methods of nervous system, in clinical neurology. Ho Chi Minh: Medical publishing house, pp. 119-147.

Le TTT (2010). Factors related to post stroke depression among older adults in Da Nang, Vietnam. PhD thesis, Burapha University.

Le VT (2004). Transcranial Doppler, in clinical neurology. Ho Chi Minh: Medical publishing house, pp. 148-151. 
Mukamal KJ, Conigrave KM, Mittleman MA, et al. (2003). Roles of drinking pattern and type of alcohol consumed in coronary heart disease in men. N. Engl. J. Med. 38(2): 109-118. DOI:10.1056/NEJMoa022095.

Nakajima SH and Chester KW (2019). Acute Ischemic Stroke. Stroke. 50: 344-418.

Ngo DV (2010). Political report of provincial party executive committee, $17^{\text {th }}$ party Congress, Prologue 2010-2015, Viet Tri, May 2011. Viet Tri, pp. 48-53.

Nguyen MH (2013). Stroke epidemiological features. Ha Noi Medical publishing house, pp. 11 - 41.

Nguyen TT and Hoang MV (2018). Non-communicable diseases, food and nutrition in Vietnam from 1975 to 2015: the burden and national response. Asia Pac. J. Clin. Nutr. 27(1): 19-28.

Phan TH (2004). Clinical and subclinical features of cerebral infarction in elderly patients in Department of Neurology, Bach Mai hospital. Second Degree Specialist thesis, Hanoi Medical University.

Roth GA, Mark D and Moran AE (2015). Global and Regional Patterns in Cardiovascular Mortality From 1990 to 2013. Circulation. 132: 1667-1678.

Sato S, Toyoda K, Uehara T, et al. (2008). Baseline IH Stroke Scale Score predicting outcome in anterior and posterior circulation strokes. Neurology. 70(24, Pt 2): 2371-2377.

Trinh VT (2011). Study on several stroke epidemiological features and efficiency of rehabilitation at home in Khanh Hoa province. $\mathrm{PhD}$ thesis in Medicine, Vietnam Military Medical University, Hanoi.

Webb AJS, Mazzucco S, Li L and Rothwell PM (2018). Prognostic significance of blood pressure variability on beat-tobeat monitoring after transient ischemic attack and stroke. Stroke. 9(1): 62-67. DOI:10.1161/STROKEAHA.117.019107.

Wolf PA, D'Agostino RB, Kannel WB, et al. (1988). Cigarette smoking as a risk factor for stroke. The Framingham Study. JAMA. 259(7): 1025-1029.

World Health Organization. (2013). A global brief on hypertension. Report from April 2013. Contract No.: WHO/DCO/WHD/2013.2.

Zhang C, Qin Y-Y, Chen Q, et al. (2014). Alcohol intake and risk of stroke: A dose- response meta-analysis of prospective studies. Int. J. Cardiol. 174(3): 669-677. DOI: 10.1016/j.ijcard.2014.04.225. 